\title{
BMA
}

\section{Glucocorticoid receptor expression in the tonsils of children with obstructive sleep apnea hypopnea syndrome}

\author{
X. Chen and J.R. Li \\ Department of Otolaryngology-Head and Neck Surgery, Navy General Hospital, \\ Beijing, China \\ Corresponding author: J.R. Li \\ E-mail: lijinrang322@163.com
}

Genet. Mol. Res. 15 (1): gmr.15017361

Received August 4, 2015

Accepted November 13, 2015

Published March 24, 2016

DOI http://dx.doi.org/10.4238/gmr.15017361

ABSTRACT. The expression of glucocorticoid receptor- $\alpha$ and $-\beta$ (GR- $\alpha$ and GR- $\beta$ ) in the tonsil tissues of children with and without obstructive sleep apnea hypopnea syndrome (OSAHS) was evaluated. A total of 30 children with OSAHS who underwent tonsillectomy in the Navy General Hospital from June 2012 to June 2014 were enrolled as the experimental group, and 30 non-OSAHS children were enrolled as the control group. The diagnosis of OSAHS was confirmed by preoperative sleep monitoring. The expression of GR- $\alpha$ and GR- $\beta$ in tonsil tissues was detected using western blot and immunohistochemical analyses. GR- $\alpha$ and GR- $\beta$ were both expressed in the tonsil tissues of OSAHS and non-OSAHS patients. The expression of GR- $\alpha$ in the tonsil tissues of OSAHS children was significantly lower than that in the tonsil tissues of non-OSAHS children, while the expression of GR- $\beta$ in the tonsil tissues was similar between the two groups of children. Since it has been reported that GR- $\alpha$ expression is correlated to glucocorticoid therapy sensitivity, the sensitivity of children with OSAHS to glucocorticoid treatment may be lower than that in children 
who do not have OSAHS. However, the function of GR in children with OSAHS still requires further investigation.

Key words: Obstructive sleep apnea hypopnea syndrome; Tonsil; Glucocorticoid receptor; Glucocorticoids

\section{INTRODUCTION}

Obstructive sleep apnea hypopnea syndrome (OSAHS) in children refers to partial or total airway obstruction that frequently occurs during sleep, and interferes with the normal ventilation and sleep structure, causing a series of pathophysiological changes that result in damage to the nervous, circulatory, and respiratory systems (Garetz et al., 2015). Tonsillar hypertrophy is one of the major causes of OSAHS in children. Hypertrophy of the tonsils causes increased resistance in the upper airway during inhalation, which generates negative pressure in the pharynx and brings the soft palate and tongue root closer to the posterior pharyngeal wall. The impact of tonsillar hypertrophy is more obvious in the supine position, where it can cause dyspnea during sleep and mouth breathing in children. Patients with tonsillar hypertrophy have oropharyngeal dryness and reduced local immunity, and are prone to upper respiratory tract infections. Recurrent upper respiratory tract infections further cause pharyngeal lymphoid hyperplasia, which results in further hypertrophy of the tonsils and exacerbation of the dyspnea. Repeated respiratory infections also cause continuous tonsillar hypertrophy, and therefore OSAHS symptoms are also exacerbated accordingly and are difficult to alleviate (Ngiam and Cistulli, 2015).

Currently, tonsillectomy is the most common treatment for OSAHS in children. However, surgical procedures come with a risk of many complications such as infection, pain, and hemorrhage. Additionally, many patients still experience sleep-disordered breathing after surgery (Chervin et al., 2015). Therefore, identifying effective drug treatment options for OSAHS in children is important to prevent surgery-associated risks. An increasing number of studies in recent years have indicated that local or systemic glucocorticoids can effectively treat OSAHS (Zhang and Xiao, 2012). However, the specific mechanism of action remains unclear. In the current study, western blot and immunohistochemical analyses were used to detect the expression of the glucocorticoid receptor (GR)- $\alpha$ and GR- $\beta$ in tonsil tissues to provide a theoretical basis for the clinical application of glucocorticoids to treat OSAHS in children.

\section{MATERIAL AND METHODS}

\section{Patient information}

A total of 30 non-OSAHS patients and 30 children with OSAHS who received inpatient surgical treatment at the Navy General Hospital from June 2012 to June 2014 were enrolled in this study. Of the 30 OSAHS patients, there were 20 males and 10 females whose ages were between 3 and 14 years, with a median age of 7.9 years. This study was approved by the Ethics Committee of our hospital. The legal guardians of all study participants provided written informed consent prior to surgery. Preoperative monitoring confirmed the diagnosis of OSAHS in 30 children, among which the apnea-hypopnea index (AHI) was $13.51 \pm 9.34$ times/h, and the average apnea index (Al) was $6.54 \pm 8.12$ times/h. Of the 30 non-OSAHS patients, there were 19 males and 11 females with an age range of 3-13 years, and a median age of 7.7 years. For these patients, the AHI was 
$1.62 \pm 0.92 \mathrm{times} / \mathrm{h}$, and the Al was $6.5 \pm 8.1$ times/h. The inclusion criterion necessitated that the patients were undergoing surgical treatment for the first time. Patients who had received antibiotics or glucocorticoid treatment in the 3 weeks prior to surgery were excluded.

\section{Western blot analysis of GR $\alpha$ and GR $\beta$ expression}

Specimens of freshly resected tonsil tissue (approximately $100 \mathrm{mg}$ ) were fully homogenized using protein lysis buffer (Beyotime, Nantong, China) and centrifuged at 12,000 rpm for $10 \mathrm{~min}$. The supernatants were collected, and the protein concentrations were determined using the bicinchoninic acid method. Appropriate amounts of protein loading buffer (Beyotime) were added based on protein concentrations, and the samples were boiled for $10 \mathrm{~min}$. Next, 35$\mu \mathrm{g}$ protein samples were electrophoresed and transferred to membranes. After blocking in $5 \%$ skim milk, the membranes were incubated with anti- $\beta$-actin, GR- $\alpha$, or GR- $\beta$ antibodies (Santa Cruz Biotechnology, Dallas, TX, USA) diluted $1: 200$ in $5 \%$ milk at $4^{\circ} \mathrm{C}$ overnight. The membranes were washed with Tris-buffered saline with Tween-20 (TBST), and then incubated with horseradish peroxidase-labeled rabbit anti-goat secondary antibodies diluted in $5 \%$ milk. The membranes were washed with TBST, and a 1:1 chemiluminescence working solution was prepared according to the enhanced chemiluminescence manual. The membranes were visualized using a gel imager, and the ImageJ software (NIH, Bethesda, MD, USA) was used to analyze band density.

\section{Immunohistochemical staining}

The resected tissue specimens were fixed in $10 \%$ formalin, conventionally embedded in paraffin, sectioned at $4 \mu \mathrm{m}$, heated to $60^{\circ} \mathrm{C}$ for $8 \mathrm{~h}$, and stored until further use. The paraffin sections were later deparaffinized, rehydrated, and washed 3 times with phosphate-buffered saline (PBS) for 3 min per wash. Next, the sections were immersed in distilled water, and antigen retrieval was conducted using ethylenediaminetetraacetic acid buffer at $100^{\circ} \mathrm{C}$ for $20 \mathrm{~min}$. After cooling to room temperature, the sections were washed twice with distilled water and twice with PBS for 3 min per wash. Peroxidase solution was added dropwise onto the sections, and the mixture was incubated at room temperature for $10 \mathrm{~min}$ to block endogenous peroxidase activity. The sections were then washed 3 times with PBS for 10 min per wash. Next, non-immunized animal serum was added dropwise onto the sections, and the mixture was incubated at room temperature for $10 \mathrm{~min}$. The solution was replaced with primary anti-GR- $\alpha$ and anti-GR- $\beta$ antibodies (Santa Cruz Biotechnology) at a 1:50 dilution in PBS, and then incubated at room temperature for $60 \mathrm{~min}$. Negative controls were incubated with PBS only. After washing 3 times with PBS for 3 min per wash, biotinylated goat anti-rabbit IgG secondary antibodies (ZSGB-BIO, Beijing, China) were added dropwise onto the sections, and the mixture was incubated at room temperature for $10 \mathrm{~min}$. After washing 3 times with PBS for 10 min per wash, avidin peroxidase solution was added dropwise onto the sections, which were then incubated at room temperature for $10 \mathrm{~min}$. After washing 3 times with PBS for 3 min per wash, the sections were developed using the 3,3'-diaminobenzidine reagent. After washing with tap water, the sections were counterstained with hematoxylin. Bluing of the sections was conducted using tap water, and then the sections were hydrated using ethanol, cleared using xylene, and mounted using neutral balsam. The immunohistochemical results were interpreted by two experienced pathologists using a double-blind method. Cells were counted in 15 fields per section at a high magnification (400X). The average number of GR- $\alpha$ - or GR- $\beta$-positive cells was used as the final result for each patient. 


\section{Statistical analysis}

Statistical analyses were performed using the SPSS 17.0 software (SPSS Inc., Chicago, IL, USA). Comparisons between two groups were conducted using Student $t$-tests. $\mathrm{P}<0.05$ was considered statistically significant.

\section{RESULTS}

\section{GR- $\alpha$ and GR- $\beta$ expression in OSAHS and non-OSAHS children}

As shown in Figure 1 and Table 1, the western blot results showed that the expression level of GR- $\alpha$ in the tonsil tissues of OSAHS children was significantly lower than that in nonOSAHS children $(P<0.05)$, while the expression of GR- $\beta$ in the tonsil tissues of children in both groups was not significantly different $(P>0.05)$.

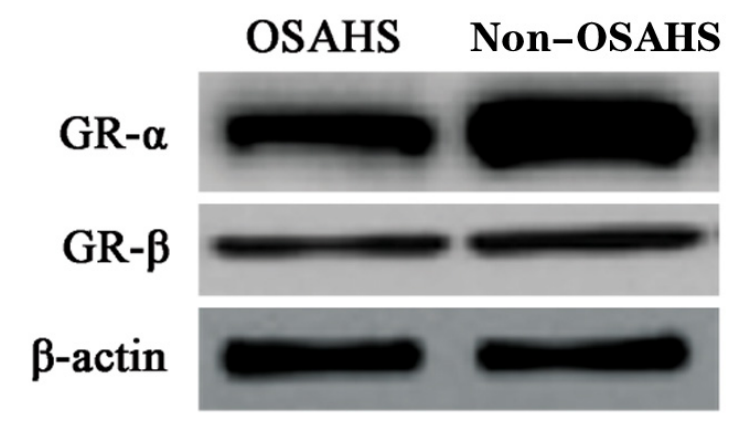

Figure 1. Detection of GR- $\alpha$ and GR- $\beta$ expression in OSAHS and non-OSAHS children using western blot analysis.

Table 1. Western blot and immunohistochemical analysis results of GR- $\alpha$ and GR- $\beta$ expression in OSAHS and non-OSAHS children.

\begin{tabular}{l|c|c|c|c|c}
\hline \multirow{2}{*}{ Group } & \multirow{2}{*}{$\mathrm{N}$} & \multicolumn{2}{|c|}{ Western blot results (protein/ $\beta$-actin) } & \multicolumn{2}{c}{$\begin{array}{c}\text { Immunohistochemical results } \\
\text { (number/400X magnification) }\end{array}$} \\
\cline { 3 - 6 } & & GR- $\alpha$ & GR- $\beta$ & GR- $\alpha$ & GR- $\beta$ \\
\hline OSAHS & 30 & $1.01 \pm 0.15$ & $0.53 \pm 0.11$ & $134 \pm 17$ & $41 \pm 6$ \\
\hline Non-OSAHS & 30 & $2.59 \pm 0.63$ & $0.51 \pm 0.15$ & $205 \pm 32$ & $43 \pm 7$ \\
\hline$t$ value & & 324.9 & 0.59 & 10.73 & 1.19 \\
\hline P value & & $<0.001$ & 0.10 & $<0.001$ & 0.41 \\
\hline
\end{tabular}

\section{Immunohistochemical analysis of GR- $\alpha$ and GR- $\beta$ expression}

The immunohistochemistry results showed that cells that were positive for GR- $\alpha$ and GR- $\beta$ stained brown-yellow (Figure 2 and Table 1). Additionally, GR- $\alpha$ and GR- $\beta$ were both expressed in the OSAHS group and in the non-OSAHS group. The number of GR- $\alpha$-positive cells in the OSAHS group was significantly lower than that in the non-OSAHS group (Figure 2, $\mathrm{P}<0.05$ ), while the number of GR- $\beta$-positive cells was not significantly different between the two groups (Table $1, \mathrm{P}>$ 0.05). These results were in agreement with the western blot results. 

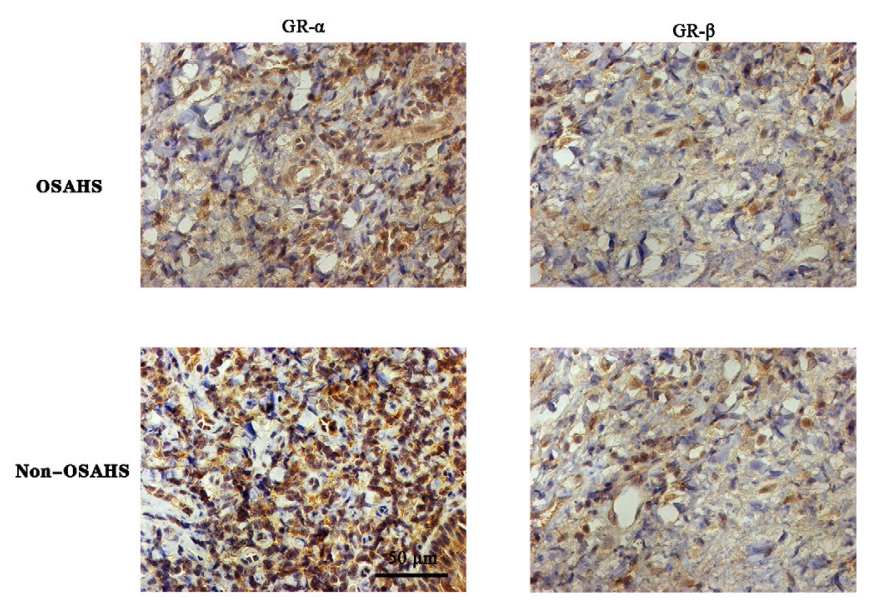

Figure 2. Detection of GR- $\alpha$ and GR- $\beta$ expression in OSAHS and non-OSAHS children using immunohistochemical analysis.

\section{DISCUSSION}

GR is a member of the conserved nuclear receptor superfamily, and is composed of the following 3 functional domains: N-terminal transcription activation domain, central DNA-binding domain, and C-terminal GR binding domain (Quax et al., 2013). Under normal physiological conditions, GR is primarily localized in the cytoplasm and exists as two isoforms, the primary GR- $\alpha$ isoform and the GR- $\beta$ isoform. GR- $\alpha$ is expressed in almost all tissues and cells, and its levels are significantly higher than those of GR- $\beta$. Hence, glucocorticoids exert their biological effects primarily through binding to GR- $\alpha$, thereby enabling the ligand-receptor complex to bind specifically to the promoter regions of target genes to regulate their activity (Kadmiel and Cidlowski, 2013; Bazsó et al., 2015). GR- $\beta$ does not bind glucocorticoids and mainly interacts with DNA. However, recent studies have shown that GR- $\beta$ has antagonistic effects on GR- $\alpha$ (Fardet and Fève, 2014). GR expression is critical for glucocorticoids to exert their effects. Many clinical studies have indicated that the density of GR- $\alpha$ and its affinity for glucocorticoids is positively correlated with glucocorticoid therapy efficacy. In elderly patients with severe asthma, the affinity between GR- $\alpha$ and glucocorticoids in those who were insensitive to glucocorticoid therapy was significantly lower than in those who were sensitive to glucocorticoid therapy or in normal controls (Oakley and Cidlowski, 2013). Studies on interstitial lung disease patients showed that the GR- $\alpha$ density in patients who were insensitive to glucocorticoid therapy was significantly lower than in patients who were sensitive to glucocorticoid therapy, further suggesting that the expression level of GR- $\alpha$ plays an important role in the sensitivity to glucocorticoid therapy (Caratti et al., 2015). However, studies on GR- $\alpha$ expression in the tonsil tissues of OSAHS children are still lacking. Therefore, in-depth studies on the expression of GR- $\alpha$ in the tonsil tissues of OSAHS children have an important role in guiding the use of glucocorticoid therapy in these children.

Previous studies have shown that the expression level of GR- $\alpha$ in adenoid tissues of OSAHS children was significantly higher than that in non-OSAHS children (Zhang et al., 2007). However, currently, there are few relevant reports on the expression levels of GR- $\alpha$ and GR- $\beta$ in the tonsil tissues of OSAHS children. In the present study, we used western blot and immunohistochemical analyses to demonstrate that GR- $\alpha$ and GR- $\beta$ were both expressed in the tonsil tissues of OSAHS 
and non-OSAHS children. The GR- $\alpha$ expression level in the tonsil tissues of OSAHS children was significantly lower than that in non-OSAHS children, while the expression level of GR- $\beta$ in the tonsil tissues of children in both groups was not significantly different. These results were consistent with previous reports on the expression levels of GR- $\alpha$ and GR- $\beta$ in the adenoid tissues of OSAHS and non-OSAHS children. Therefore, taken together with previous studies, the results herein suggest that the sensitivity of OSAHS children to glucocorticoid therapy may be lower than the sensitivity of non-OSAHS children.

In summary, GR- $\alpha$ and GR- $\beta$ were both expressed in the tonsil tissues of OSAHS children. Their expression levels may be an important factor affecting the sensitivity of OSAHS children to glucocorticoid therapy. However, these results require further validation in in vitro cell culture experiments and animal models.

\section{Conflicts of interest}

The authors declare no conflict of interest.

\section{ACKNOWLEDGMENTS}

The authors wish to express their gratitude to the reviewers for their critical comments.

\section{REFERENCES}

Bazsó A, Szappanos Á, Patócs A, Poór G, et al. (2015). The importance of glucocorticoid receptors in systemic lupus erythaematosus. A systematic review. Autoimmun. Rev. 14: 349-351.http://dx.doi.org/10.1016/j.autrev.2014.12.007

Caratti G, Matthews L, Poolman T, Kershaw S, et al. (2015). Glucocorticoid receptor function in health and disease. Clin. Endocrinol. 83: 441-448.http://dx.doi.org/10.1111/cen.12728

Chervin RD, Ellenberg SS, Hou X, Marcus CL, et al. (2015). Prognosis for Spontaneous Resolution of Obstructive Sleep Apnea in Children. Chest; Epub Ahead of Print. http://dx.doi.org/10.1378/chest.14-2873

Fardet $L$ and Fève B (2014). Systemic glucocorticoid therapy: a review of its metabolic and cardiovascular adverse events. Drugs 74: 1731-1745.http://dx.doi.org/10.1007/s40265-014-0282-9

Garetz SL, Mitchell RB, Parker PD, Moore RH, et al. (2015). Quality of life and obstructive sleep apnea symptoms after pediatric adenotonsillectomy. Pediatrics 135: e477-e486.http://dx.doi.org/10.1542/peds.2014-0620

Kadmiel M and Cidlowski JA (2013). Glucocorticoid receptor signaling in health and disease. Trends Pharmacol. Sci. 34: 518530.http://dx.doi.org/10.1016/j.tips.2013.07.003

Ngiam J and Cistulli PA (2015). Dental treatment for paediatric obstructive sleep apnea. Paediatr. Respir. Rev. 16: 174-181. http://dx.doi.org/10.1016/j.prrv.2014.11.002

Oakley RH and Cidlowski JA (2013). The biology of the glucocorticoid receptor: new signaling mechanisms in health and disease. J. Allergy Clin. Immunol. 132: 1033-1044.http://dx.doi.org/10.1016/j.jaci.2013.09.007

Quax RA, Manenschijn L, Koper JW, Hazes JM, et al. (2013). Glucocorticoid sensitivity in health and disease. Nat. Rev. Endocrinol. 9: 670-686.http://dx.doi.org/10.1038/nrendo.2013.183

Zhang HK and Xiao KL (2012). Progressions on the studies on the treatment of obstructive sleep apnea hypopnea syndrome in children with intranasal corticosteroids. Zhongguo Yan Er Bi Hou Ke Za Zhi 12: 266-268.

Zhang XW, Li Y, Xie MQ, Zhang GH, et al. (2007). [Glucocorticoid receptor subunit expression in adenoid tissue of children with obstructive sleep apnea hypopnea syndrome]. Zhonghua Er Bi Yan Hou Tou Jing Wai Ke Za Zhi 42: 109-112. 